\title{
Análise de metais pesados em amostras de Peumus boldus Mol. (Monimiaceae)
}

\author{
Melissa Schwanz, ${ }^{1}$ Jéferson J. Ferreira, ${ }^{2}$ Pedro Fröehlich, ${ }^{1}$ José A. S. Zuanazzi, ${ }^{1}$ \\ Amélia T. Henriques $*$,
}

\author{
'Programa de Pós-Graduação em Ciências Farmacêuticas, Faculdade de Farmácia, Universidade Federal do \\ Rio Grande do Sul, Av. Ipiranga 2752, 90610-000 Porto Alegre-RS, Brasil, \\ ${ }^{2}$ Laboratório de Química Farmacêutica, Universidade Federal do Rio Grande do Sul, Av. Ipiranga 2752, \\ 90610-000 Porto Alegre-RS, Brasil
}

\begin{abstract}
RESUMO: Oito amostras, provenientes do Brasil, Chile e Argentina, de Peumus boldus Molina (Monimiaceae), espécie comum e abundante no Chile, cujas folhas são amplamente empregadas pela medicina tradicional para o tratamento de uma variedade de afecções do sistema digestivo e hepatobiliar, foram analisadas, após digestão nítrica, para a quantificação de ferro, manganês, cobre, chumbo, cromo, cobalto e níquel, por espectrofotometria de absorção atômica. Chumbo, cromo e cobalto não foram detectados (limite de detecção de $5 \mu \mathrm{g} / \mathrm{g}$ ) em nenhuma das amostras. Todas as amostras apresentaram maior teor em ferro, que variou de $109,7 \mathrm{mg} / \mathrm{kg}$ a $315,7 \mathrm{mg} / \mathrm{kg}$, seguido por manganês $(65,5 \mathrm{mg} / \mathrm{kg}$ a $158,8 \mathrm{mg} / \mathrm{kg})$, cobre $(3,04 \mathrm{mg} / \mathrm{kg}$ a $9,16 \mathrm{mg} / \mathrm{kg})$ e níquel $(0,77 \mathrm{mg} / \mathrm{kg}$ a $4,31 \mathrm{mg} / \mathrm{kg})$.
\end{abstract}

Unitermos: Peumus boldus, metais pesados, absorção atômica.

\begin{abstract}
Analysis of heavy metals in samples of Peumus boldus Mol. (Monimiaceae)". Eight samples, obtained from Brazil, Chile and Argentina, of Peumus boldus Molina (Monimiaceae), an abundant and widespread native tree in Chile, which leaves are widely used in folk medicine for the treatment of digestive and hepatobiliary disorders, were analyzed, after nitric digestion, for the content of iron, manganese, copper, lead, chromium, cobalt and nickel, by atomic absorption spectrophotometry. Lead, chromium and cobalt were not detected (detection limit of $5 \mu \mathrm{g} / \mathrm{g}$ ) in any sample. The samples showed a high level of iron, which ranged from 109.7 $\mathrm{mg} / \mathrm{kg}$ to $315.7 \mathrm{mg} / \mathrm{kg}$, followed by manganese $(65.5 \mathrm{mg} / \mathrm{kg}$ to $158.8 \mathrm{mg} / \mathrm{kg})$, copper $(3.04 \mathrm{mg} /$ $\mathrm{kg}$ to $9.16 \mathrm{mg} / \mathrm{kg})$ and nickel $(0.77 \mathrm{mg} / \mathrm{kg}$ to $4.31 \mathrm{mg} / \mathrm{kg})$.
\end{abstract}

Keywords: Peumus boldus, heavy metals, atomic absorption.

\section{INTRODUÇ̃̃O}

A utilização de espécies vegetais com propósito terapêutico tem relatos milenares, porém só mais recentemente os cientistas procuram avaliar a extensão dos benefícios terapêuticos e segurança com seu uso, principalmente devido a relatos de graves efeitos colaterais e fatalidades, algumas destas associadas à presença de metais tóxicos (Tyler, 1987; Kakosy et al., 1996; Stewart et al., 1999; Ernst, 2002).

Os metais pesados constituem a maior fonte poluidora inorgânica de solos e águas, sendo introduzidos no ambiente, principalmente através de fertilizantes, pesticidas, combustão de carvão e óleo, emissões veiculares, mineração, fundição, refinamento e incineração de resíduos urbanos e industriais (Tavares \& Carvalho, 1992).

As plantas podem acumular estes metais em todos os tecidos, podendo transferi-los para a cadeia alimentar, e esta acumulação é um dos temas de interesse ambiental atualmente, não apenas pela fitotoxicidade de muitos destes metais, mas também pelos potenciais efeitos nocivos na saúde animal e humana (Maiga et al., 2005).

Alguns metais são considerados essenciais ao desenvolvimento de plantas ou animais, porém, os limites de tolerância a estes microelementos, de maneira geral, são muito baixos. Dentre eles destacamos o ferro (Fe), elemento vital para a maioria dos organismos vivos, participando de uma série de processos metabólicos; o cobalto $(\mathrm{Co})$, necessário para a formação da vitamina B12; o cobre $(\mathrm{Cu})$, componente essencial de enzimas como a tirosinase, citocromo oxidase e superóxido dismutase; o manganês $(\mathrm{Mn})$, presente em metaloproteínas como a piruvato descarboxilase; o cromo $(\mathrm{Cr})$, elemento necessário para o metabolismo do colesterol, gordura e glicose; e o níquel (Ni), que participa da regulação de lipídeos e síntese de fosfolipídeos (Klassen \& Watkins, 2001).

Outros são considerados exclusivamente 
tóxicos, como o chumbo $(\mathrm{Pb})$, conhecido por induzir várias disfunções em animais de laboratório e humanos, alterando a atividade antioxidante por inibição do grupo funcional $\mathrm{SH}$ em muitas enzimas. No entanto, todos os elementos em altas concentrações podem trazer danos ao organismo, por interagirem diretamente com o DNA (Maiga et al., 2005; Klassen \& Watkins, 2001; Hsu \& Guo, 2002).

$\mathrm{Na}$ literatura encontram-se vários relatos de quantificação de metais pesados em plantas medicinais e amostras de alimentos (Rajurkar \& Damane, 1998; Abou-Arab et al., 1999; Lopes et al., 2002; Macari et al., 2002; Bordajandi et al., 2004; Caldas \& Machado, 2004; Kannamkumarath et al., 2004; Palmieri et al., 2005; Delaporte et al., 2005). Desta forma, o objetivo deste trabalho foi a quantificação de sete microelementos (Fe, $\mathrm{Mn}, \mathrm{Cu}, \mathrm{Pb}, \mathrm{Cr}, \mathrm{Co}$ e Ni) em amostras obtidas em indústrias e comércio do Brasil, Chile e Argentina de folhas de Peumus boldus Mol. (Monimiaceae). O boldo representa um caso de planta medicinal na qual o uso é intensamente fundamentado na medicina tradicional e que há muito vem sendo objeto de considerável atenção do ponto de vista farmacológico e químico (Speisky \& Cassels, 1994; Melo et al., 2004; Tôrres et al., 2005; Mendes et al., 2006; Agra et al., 2007). A importância dessa planta levou sua inclusão na primeira, segunda e quarta edições da Farmacopéia Brasileira (Brandão et al., 2006).

\section{MATERIAIS E MÉTODOS}

\section{Amostras}

As amostras de Peumus boldus utilizadas, bem como o ano e local de obtenção do material estão listados na Tabela 1.

\section{Preparação do material}

Cerca de $20 \mathrm{mg}$ de amostra foram dissolvidas em $1 \mathrm{~mL}$ de ácido nítrico P.A. (Merck) previamente bidestilado para retirada de qualquer impureza inorgânica. Esta solução foi levada a banho de ultra-som durante 30 minutos e, em seguida, colocada em estufa $\left(60{ }^{\circ} \mathrm{C}\right)$ durante uma hora. $\mathrm{O}$ sobrenadante foi então diluído em água ultrapura até atingir as concentrações da curva padrão. Três diluições foram preparadas e analisadas para cada amostra.

\section{Análise por espectrofotômetro de absorção atômica}

As concentrações dos elementos foram determinadas em um aparelho Espectrofotômetro de Absorção Atômica Perkin Elmer Analist - 300, equipado com forno de grafite HGA - 800 e amostrador AS - 70. Foram utilizadas lâmpadas monoelemento de cátodo oco para as análises. As concentrações das amostras analisadas foram calculadas com base na leitura de quatro diferentes concentrações das soluções padrão do elemento em questão.

\section{RESULTADOS E DISCUSSÃO}

As concentrações de metais encontradas nas amostras de Peumus boldus estão apresentadas na Tabela 2 e Figura 1. Todas as amostras foram analisadas em triplicata.

A concentração de metais pesados neste estudo decresceu na ordem: $\mathrm{Fe}>\mathrm{Mn}>\mathrm{Cu}>\mathrm{Ni}$, sendo que $\mathrm{Pb}$, $\mathrm{Cr}$ e Co tiveram níveis de quantificação abaixo do limite de detecção de $5 \mu \mathrm{g} / \mathrm{g}$.

Todas as amostras apresentaram níveis mais elevados de Fe comparado aos outros elementos, que variaram de $109,7 \mathrm{mg} / \mathrm{kg}$ a $315,7 \mathrm{mg} / \mathrm{kg}$. O limite tolerável de Fe, segundo Maiga et al. (2005) é de $45 \mathrm{mg}$. Isto corresponderia a aproximadamente $142 \mathrm{~g}$ de folhas de Peumus boldus da amostra 6, ou $410 \mathrm{~g}$ de folhas da amostra 3, ambas amostras obtidas no Brasil. Duas amostras adquiridas no Brasil apresentaram o menor teor.

$\mathrm{O} \mathrm{Mn}$ foi o segundo microelemento de maior concentração quantificado nas amostras. Seus níveis variaram de $158,8 \mathrm{mg} / \mathrm{kg}$ a $65,5 \mathrm{mg} / \mathrm{kg}$. A recomendação diária de $\mathrm{Mn}$ na dieta é de 2 a $5 \mathrm{mg}$ de $\mathrm{Mn}^{2+}$, correspondendo a aproximadamente, 12,59 g a $31,48 \mathrm{~g}$ da amostra 4; e 30,53 $\mathrm{g}$ a 76,34 $\mathrm{g}$ da amostra 7 (Maiga et al., 2005).

Os níveis de $\mathrm{Cu}$ variaram de 9,16 mg/kg a 3,04 $\mathrm{mg} / \mathrm{kg}$. Todos os valores encontrados ficaram abaixo do limite permitido pela OMS em alimentos, que é de 4 $\mathrm{mg} / 100 \mathrm{~g}$. Conforme Portaria 685 da ANVISA (Brasil, 1998), os níveis toleráveis de $\mathrm{Cu}$ em alimentos variam de $0,1 \mathrm{mg} / \mathrm{kg}$ a $10 \mathrm{mg} / \mathrm{kg}$. Nenhum dos valores obtidos na análise fica abaixo do nível de $0,1 \mathrm{mg} / \mathrm{kg}$, no entanto, este limite corresponde a óleos, gorduras e emulsões refinadas (Brasil, 1998; Maiga et al., 2005). A presença deste elemento pode estar relacionada com o tipo de solo na região de cultivo de todas as amostras, o Chile. Destaca-se nesta análise a amostra número 8 adquirida diretamente do país produtor a qual pode ter sido cultivada em região com solo mais rico neste elemento.

Os níveis de $\mathrm{Ni}$ variaram de $4,31 \mathrm{mg} / \mathrm{kg}$ a 0,77 $\mathrm{mg} / \mathrm{kg}$. Estes valores encontrados não ultrapassam a recomendação diária deste metal mesmo para crianças lactentes, que é de 5 a $15 \mu \mathrm{g} / \mathrm{g}$ de Ni por dia no leite (Barceloux, 1999).

Os microelementos $\mathrm{Pb}, \mathrm{Cr}$ e Co tiveram níveis de detecção abaixo de $5 \mu \mathrm{g} / \mathrm{g}$. Conforme a OMS, o limite máximo permitido para alimentos de chumbo é de $10 \mu \mathrm{g} / \mathrm{g}$, enquanto que a Portaria 685 da ANVISA (Brasil, 1998) tolera limites de $0,05 \mathrm{mg} / \mathrm{kg}$ a $2,0 \mathrm{mg} /$ $\mathrm{kg}$. A Farmacopéia Européia indica um limite máximo de chumbo em plantas medicinais de $5 \mathrm{mg} / \mathrm{kg}$. Para o cromo a recomendação diária prescrita no FDA para 
Tabela 1. Lista de amostras utilizadas na realização do trabalho.

\begin{tabular}{|c|c|c|c|c|}
\hline $\mathrm{N}^{\mathrm{o}}$ & Local de obtenção & & & Ano \\
\hline 1 & $\begin{array}{llll}\text { Indústria } & \text { Farmacêutica } & \text { A } & \text { (Porto } \\
\text { Brasil) } & & & \\
\end{array}$ & Alegre & & 2004 \\
\hline 2 & Ervanário (Manaus - Brasil) & & & 2004 \\
\hline 3 & $\begin{array}{l}\text { Indústria Farmacêutica } \mathrm{B} \text { (Porto } \\
\text { Brasil) }\end{array}$ & Alegre & 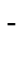 & 2004 \\
\hline 4 & Comércio (Buenos Aires - Argentina & & & 2004 \\
\hline 5 & Comércio (Buenos Aires - Argentina & & & 2004 \\
\hline 6 & Comércio (Porto Alegre - Brasil) & & & 2005 \\
\hline 7 & Comércio (Santiago - Chile) & & & 2004 \\
\hline 8 & Comércio (Santiago - Chile) & & & 2004 \\
\hline
\end{tabular}

$* \mathrm{mg} / \mathrm{kg} ; * * \mu \mathrm{g} / \mathrm{g}$

Tabela 2. Concentrações médias dos metais nas amostras de Peumus boldus.

\begin{tabular}{cccccccc}
\hline Amostra & $\mathrm{Fe}^{*}$ & $\mathrm{Mn}^{*}$ & $\mathrm{Cu}^{*}$ & $\mathrm{Ni}^{*}$ & $\mathrm{Co}^{* *}$ & $\mathrm{Cr}^{* *}$ & $\mathrm{~Pb}^{* *}$ \\
\hline 1 & 221,5 & 105,9 & 3,75 & 1,30 & & & \\
2 & 139,4 & 93,5 & 3,10 & 2,10 & & & \\
3 & 109,7 & 133,0 & 3,04 & 1,10 & & & \\
4 & 255,6 & 158,8 & 4,32 & 0,98 & & & \\
5 & 285,5 & 87,3 & 4,39 & 0,77 & & $<5$ & $<5$ \\
6 & 315,7 & 136,3 & 3,74 & 4,31 & & & \\
7 & 254,1 & 65,5 & 5,59 & 3,83 & & & \\
8 & 267,2 & 108,5 & 9,16 & 3,46 & & & \\
\hline
\end{tabular}

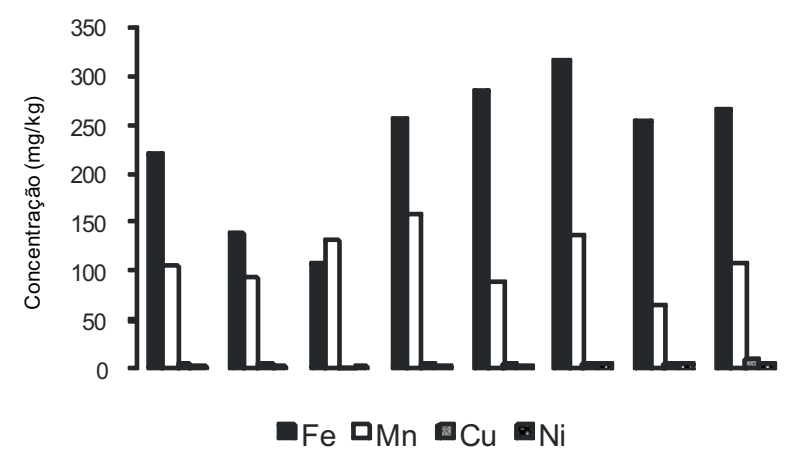

Figura 1. Comparação dos metais presentes em diferentes amostras de Peumus boldus.

alimentos é de $0,12 \mathrm{mg} / \mathrm{kg}$ (Haider et al., 2004; Brasil, 1998; European Pharmacopoeia, 2002).

Plantas absorvem íons metálicos do solo através das raízes e o aumento da concentração de metais é influenciado por vários fatores, incluindo práticas na agricultura, condições geoclimáticas, atividades antropogênicas tais como indústrias químicas, espécie da planta e a parte usada (Abou-Arab et al., 1999; Caldas \& Machado, 2004; Maiga et al., 2005). Metais pesados, principalmente o $\mathrm{Pb}$, são muito densos e solúveis em água, desta forma são facilmente absorvidos por organismos vivos (Haider et al., 2004). Todas as amostras analisadas, apesar de serem provenientes de diferentes fontes, são cultivadas na mesma região.
Assim, a inesperada variação verificada pode estar ligada a alguns dos fatores ambientais citados.

Palmieri et al. (2005) sugeriram que metais como o $\mathrm{Pb}$ são expostos no ambiente através de deposição aérea ao longo de rodovias, em proporção a densidade do tráfico, enquanto o $\mathrm{Cu}$ é acumulado por plantas particularmente pelo solo e seu conteúdo não está relacionado ao volume de tráfico.

O monitoramento de metais tóxicos é muito importante no desenvolvimento de países onde as plantas são usadas como alimentos ou com fins medicinais, como o Brasil, que possui uma elevada diversidade de plantas usadas para fins farmacológicos (Maiga et al., 2001; Caldas \& Machado, 2004).

\section{REFERÊNCIAS}

Abou-Arab AAK, Kawther MS, El Tantawy ME, Badeaa RI, Khayria N 1999. Quantity stimation of some contaminants in commonly used medicinal plants in the Egyptian market. Food Chem 67: 357-363.

Agra MF, França PF, Barbosa-Filho JM 2007. Synopsis of the plants known as medicinal and poisonous in Northeast of Brazil. Rev Bras Farmacogn 17: 114-140.

Barceloux GD 1999. Manganese, nickel. Clin Toxicol 37: 239-258 e 293-307.

Bordajandi LR, Gómez G, Abad E, Rivera J, Fernández-Baston MM, Blasco J, González J 2004. Survey of persistent organochlorine contaminants (PcBs, PCDD/FS and PAHS), heavy metals ( $\mathrm{Cu}, \mathrm{Cd}, \mathrm{Zn}, \mathrm{Pb}$ and $\mathrm{Hg}$ ), and arsenic in food samples from Huelva (Spain): levels and health implications. J Agric Food Chem 52: 992-1001.

Brandão MGL, Cosenza GP, Moreira RA, Monte-Mor RLM 2006. Medicinal plants and other botanical products from the Brazilian Official Pharmacopoeia. Rev Bras Farmacogn 16: 408-420.

Brasil 1998. Ministério da Saúde, Secretaria de Vigilância Sanitária. Portaria n. 685, de 27 de agosto de 1998. Diário Oficial da República Federativa do Brasil. D.O.U. 29 agosto 1998.

Caldas ED, Machado LL 2004. Cadmiun, mercury and lead in medicinal herbs in Brazil. Food Chem Toxicol 42: 599-603.

Delaporte RH, Guzen KP, Takemura OS, Mello JCP 2005. Estudo mineral das espécies vegetais Alternanthera brasiliana (L.) Kuntze e Bouchea fl uminensis (Vell) Mold. Rev Bras Farmacogn 15: 133-136.

Ernst E 2002.Toxic heavy metals and undeclared drugs in Asian herbal medicines. Trends Pharmacol Sci 23: 136-139.

European Pharmacopoeia 2002. 4 ed. Strasbourg: Council of Europe.

Haider S, Naithani V, Barthawal J, Kakkar P 2004. Heavy metal content in some therapeutically important medicinal plants. Bull Environ Contam Toxicol 72: 
119-127.

Hsu PC, Guo YL 2002. Antioxidant nutrients and lead toxicity. Toxicology 180: 33-44.

Kakosy T, Hudak A, Naray M 1996. Lead intoxication epidemic caused by ingestion of contamined ground paprika. J Toxicol-Clin Toxic 34: 507-511.

Kannamkumarath SS, Wuilloud RG, Caruso JA 2004. Studies of various elements of nutritional and toxicological interest associated with different molecular weight fractions in Brazil nuts. J Agric Food Chem 52: 5773-5780.

Klassen CD, Watkins III JB 2001. Toxicologia, a Ciência Básica dos Tóxicos, de Casarett e Doull. Portugal: Editora McGraw-Hill.

Lopes MFG, Almeida MMB, Nogueira CMD, Morais NMT, Magalhães CEC 2002. Estudo mineral de plantas medicinais. Rev Bras Farmacogn 12(Supl.): 115-116.

Macari PAT, Sousa RR, Crespo MLL, Martins PA, Vieira JDD, Velosa AC, Fávero O, Gomes EPC 2002. Comparação entre os metais presentes em Croton floribundus Spreng. e Baccharis dracunculifolia DC. Rev Bras Farmacogn 12(Supl. 1): 76-77.

Maiga A, Diallo D, Bye R, Paulsen BS 2005. Determination of some toxic and essential metal ions in medicinal and edible plants from Mali. J Agric Food Chem 53: -2321 .

Melo JG, Nascimento VT, Amorim ELC, Andrade Lima CS, Albuquerque UP 2004. Avaliação da qualidade de amostras comerciais de boldo (Peumus boldus Molina), pata-de-vaca (Bauhinia spp.) e ginco (Ginkgo biloba L.). Rev Bras Farmacogn 14: 111-120.

Mendes BG, Machado MJ, Falkenberg M 2006. Triagem de glicolipídios em plantas medicinais. Rev Bras Farmacogn 16: 568-575.

Palmieri RM, La Pera L, Di Bella G, Dugo G 2005. Simultaneous determination of $\mathrm{Cd}$ (II), $\mathrm{Cu}$ (II), $\mathrm{Pb}$ (II) and $\mathrm{Zn}$ (II) by derivative stripping chronopotentiometry in Pittosporum tobira leaves: a measurement of local atmosferic polluition in Messina (Sicily, Italy). Chemosphere 59: 1161-1168.

Rajurkar NS, Damane MM 1998. Mineral content of medicinal plants used in the treatment of diseases resulting from urinary tract disorders. Appl Radiat Isot 49: 773-776.

Speisky H, Cassels BK 1994. Boldo and boldine: an emerging case of natural drug development. Pharm Res 29: $1-11$.

Stewart MJ, Moar JJ, Steenkamp P, Kokot M 1999. Findings in fatal cases of poisoning attributed to traditional remedies in South Africa. Forensic Sci Int 101: 177-183.

Tavares TM, Carvalho FM 1992. Avaliação da exposição de populações humanas a metais pesados no ambiente: exemplos do Recôncavo Bahiano. Quim Nova 15: 147-153.
Tôrres AR, Oliveira RAG, Diniz MFFM, Araújo EC 2005. Estudo sobre o uso de plantas medicinais em crianças hospitalizadas da cidade de João Pessoa: riscos e benefícios. Rev Bras Farmacogn 15: 373-380.

Tyler VE 1987. Herbal medicine in America. Planta Med 14: $1-4$. 